\title{
Complete and robust bandgap switching in double-inverse-opal photonic crystals
}

\author{
Durga P. Aryal, ${ }^{\text {a) }}$ Kosmas L. Tsakmakidis, Cécile Jamois, ${ }^{\text {b) }}$ and Ortwin Hess \\ Advanced Technology Institute, Faculty of Engineering and Physical Sciences, University of Surrey, \\ Guildford GU2 7XH, United Kingdom
}

(Received 9 August 2007; accepted 4 December 2007; published online 3 January 2008)

\begin{abstract}
The authors theoretically demonstrate a practical scheme for robust and complete photonic bandgap switching using a three-dimensional double-inverse-opal photonic crystal. The investigated structure consists of a close-packed face-centered-cubic arrangement of spherical air pores, interconnected via air channels and embedded in a high-index (tin disulfide) backbone. We show that by placing lower-index movable dielectric scatterers (titania) inside the air pores, a complete photonic bandgap opens for certain positions of the scatterers, which altogether closes for other positions. Our analysis reveals that this switching scheme is robust to geometric imperfections and allows for sizeable bandgap switching. (C) 2008 American Institute of Physics. [DOI: 10.1063/1.2829789]
\end{abstract}

Since the original works of John ${ }^{1}$ and Yablonovitch, ${ }^{2}$ periodic photonic materials structured on a scale of the wavelength of light have become the subject of overwhelming research activity. Such structures, termed photonic crystals (PCs), provide a basic platform for molding the flow of light and hold promise for a breadth of future applications. Recently, the ability of PCs to control the flow of light in the visible regime has led to the emergence of a current realm of active research, wherein the aim is to use large-scale PCs to realize functional windows or displays. ${ }^{3-5} \mathrm{~A}$ characteristic example of envisioned applications is structural color (iridescent, prismatic, multihued, and luminescent), which allows for evading the environmentally unfriendly traditional dyes and is built of components which are benign, while being universally attractive in competitive marketplaces, such as mobile electronics and automotive or airline industries. Further foreseeable applications include windows that can change their appearance from transparent to opaque on demand, or that adapt their transmission properties to the direction of incident light, e.g., for more efficient solar panels. ${ }^{6}$ For such purposes, opal-based PCs are the most promising candidates since they possess three-dimensional (3D) photonic properties, and can simultaneously be fabricated on large scales and at low cost.

A central issue toward the deployment of PCs in the aforementioned applications is the extent to which tunability or even complete switching of their optical properties can be achieved. So far, researchers have primarily followed two strategies for the accomplishment of global tuning in photonic bandgap (PBG) structures. The first is, broadly, based on the control of lattice constant(s), either by means of mechanical forces ${ }^{7}$ or by volume phase transitions, ${ }^{3}$ caused by external optical illumination or by changes in the environment. While this method does allow for large shifts in the optical stop band, the associated structural changes are usually on the order of micrometers, which may be a hindrance for certain nanophotonic applications. The second methodology relies on variation of the material refractive indices,

\footnotetext{
${ }^{a)}$ Electronic mail: D.P.Aryal@surrey.ac.uk.

${ }^{b}$ Present address: Institut des Nanotechologies de Lyon, CNRS UMR5270, Université de Lyon, INSA-Lyon, Villeurbanne F-69621, France.
}

either through free-carrier injection, ${ }^{8}$ thermo-optic illumination, ${ }^{9}$ or liquid-crystal infiltration. ${ }^{10}$ While large changes in the reflection spectra have been observed in such systems, achieving complete photonic bandgap switching still remains to be shown. Moreover, the robustness of these methods to geometric imperfections and the extent to which their applicability is affected by minute or sizable fabrication deficiencies is not clear yet.

In this work, we introduce a practical scheme for achieving complete PBG switching through the collective shift of dielectric spheres placed within an opal-based PC. The investigated structure, which we call double-inverse-opal photonic crystal (DIOPC) consists of an inverse opal with highindex dielectric backbone and movable dielectric spheres inside the air pores [see Fig. 1(a)]. Such a structure has already been fabricated ${ }^{11}$ with very good homogeneity and reproducibility, which allows for large-scale applications. In this letter, we show that the DIOPC can possess a robust and completely switchable PBG of above 3\%. Moreover, this switchable PBG is independent of the size of the interconnections between the air voids, which is of great technological importance, since the size of the interconnections is the most difficult parameter to control during the fabrication process. In this way, a substantial flexibility is allowed, without any impact on the reproducibility.

A schematic description of the DIOPC structure is given in Fig. 1(a). Consider an inverse opal structure of closedpacked air pores with radius $R_{p}$, partially filled with spherical particles, and interconnected via cylindrical channels of radius $R_{\text {cyl. }}$. The background material is tin disulfide $\left(n_{b}=3.2\right)$ and the spheres are made of titania $\left(n_{\mathrm{sp}}=2.5\right)$ and have radius $R_{\mathrm{sp}}$. The principle of the fabrication process and experimental demonstration of such structures have been detailed in a series of recent works. ${ }^{11,12}$ Note that, for this specific choice of materials, the fabricated structures are transparent in most of the visible spectral range. The titania spheres have inorganic cores that are assumed to be considerably smaller than the wavelength of light. These inorganic cores can be addressed by external electric or magnetic fields, thereby allowing for a collective movement of the titania spheres at will. Details of the experimental switching process are currently under study and will be the subject of a later publica- 


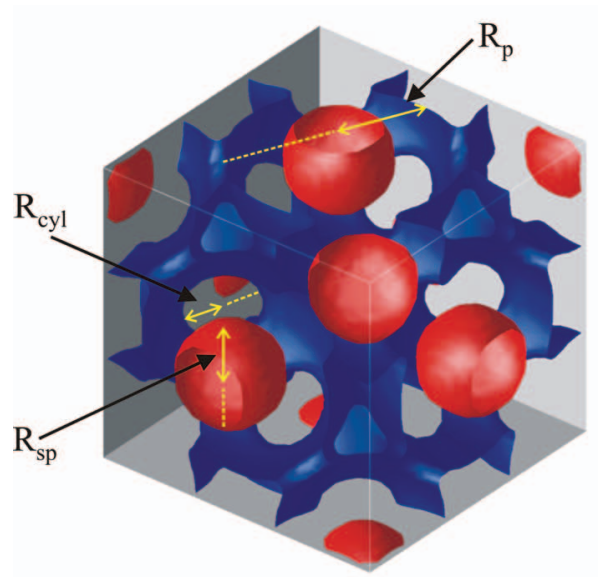

(a)

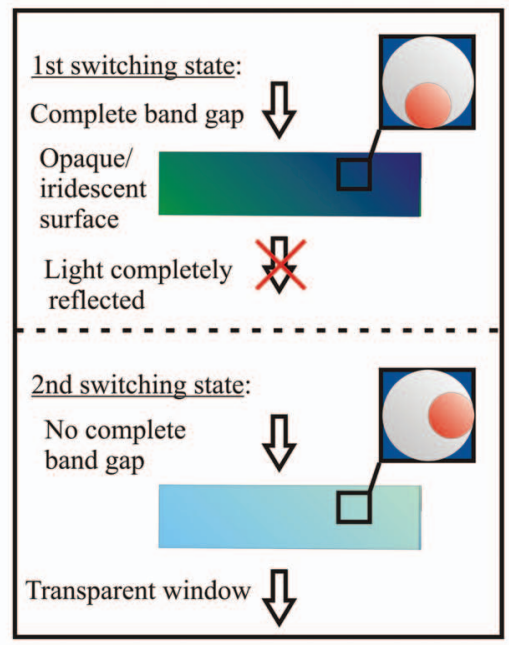

(b)

FIG. 1. (Color) (a) Illustration of an eight-unit-cell DIOPC structure along with the associated geometric definitions. Shown in red are the centred titania spheres and in blue the tin disulfide backbone material. (b) Schematic illustration of the proposed DIOPC switching mechanism.

tion. In Fig. 1(b), we summarize the principle of the switching mechanism. For one switching state [spheres in (111)], the structure presents a complete PBG, hence, incident light onto the PC cannot penetrate the structure and is entirely reflected. This kind of surface will look like a very colorful mirror. For the other switching state [spheres in (100)], there is no complete PBG, therefore, for certain directions, light can penetrate and pass through the PC. For these directions, the surface will be transparent. This phenomenon can be used for transparent/opaque switchable surfaces or, in the case of pixellization, for more complex devices, such as displays.

The optical properties of the DIOPC structure have been simulated using $a b$ initio band structure calculations, on the basis of high-accuracy plane-wave expansions of the magnetic field components on a reciprocal lattice, combined with appropriate periodic boundary conditions. ${ }^{13}$ It is to be noted that the shifting of the spheres from their central position breaks some symmetries of the PC structure; hence, some high-symmetry points are not equivalent any more. This effect is taken into account in the presented simulations when determining the position of the complete bandgap. Figure 2(a) reports the band diagram of the DIOPC structure, with Downloaded 30 Mar 2009 to 131.227.178.132. Redistribution subject

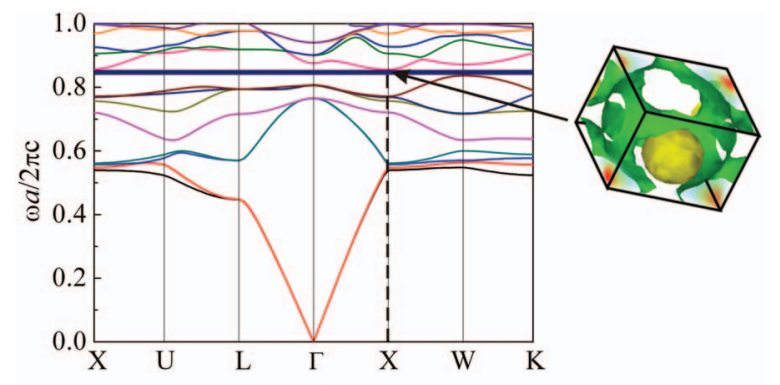

(a)

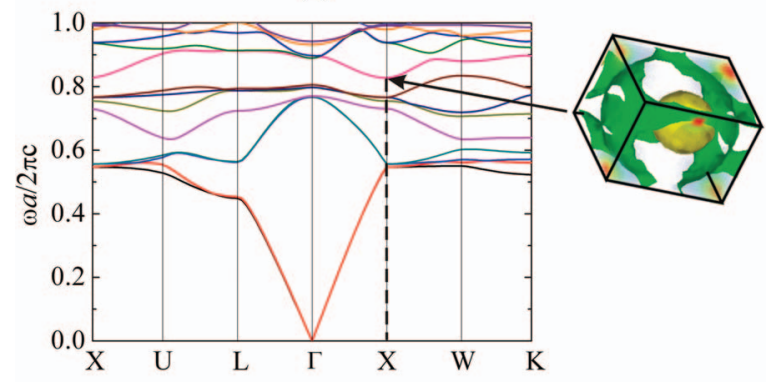

(b)

FIG. 2. (Color) Band structure of the DIOPC, with the titania core spheres shifted along the (a) (111) and (b) (100) directions. Shown on the right hand side of the two band structures are the unit cell 3D energy density distributions, which correspond to the $X$ point of the ninth band(s). Here, red color indicates high energy density values, while pale blue color designates low energy density values. The tin disulfide background (shown in green) has refractive index $n_{b}=3.2$. The core sphere (shown in yellow) and cylinder radii are chosen to be $0.13 a$ and $0.08 a$, respectively. The pore radius is $0.354 a$.

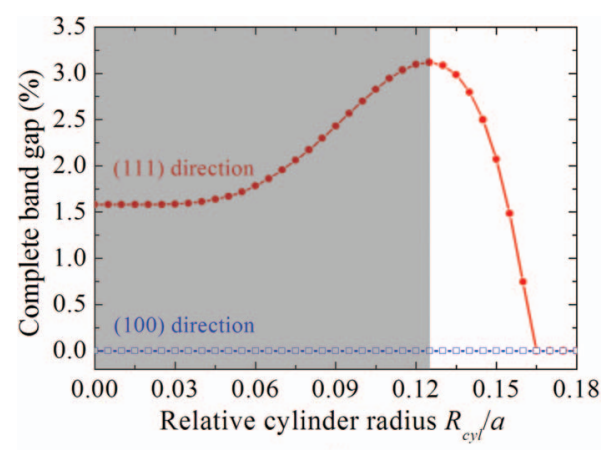

(a)

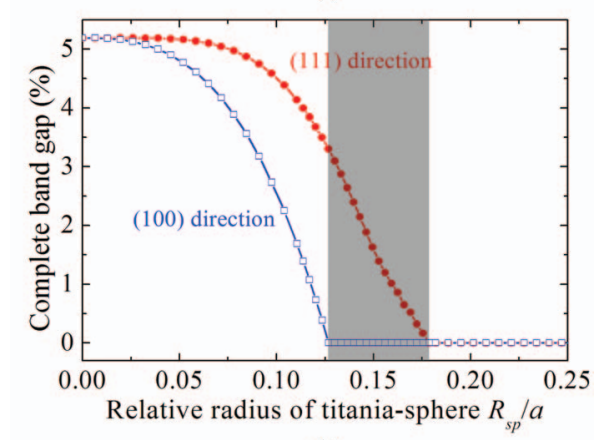

(b)

FIG. 3. (Color) Variations of the complete bandgap for two switching states: spheres shifted in the (111) (red) and the (100) (blue) direction. (a) Dependence of the bandgap size on $R_{\mathrm{cyl}}$, for $R_{\mathrm{sp}}=0.13 a$. (b) Dependence of the bandgap size on $R_{\mathrm{sp}}$, for $R_{\mathrm{cyl}}=0.12 a$. Parameter ranges in which a complete switching is experimentally feasible are highlighted by the gray shaded areas.
to AlP license or copyright; see http://apl.aip.org/apl/copyright.jsp 
the titania spheres being in their "natural" (111) position. For simplicity, the presence of the small inorganic inclusions is not taken into account in these calculations. The radii of the core, cylinder, and air pores are $R_{\mathrm{sp}}=0.13 a, R_{\mathrm{cyl}}=0.08 a$, and $R_{p}=0.354 a$, respectively, with $a$ being the (fcc) lattice constant of the crystal. One can observe from Fig. 2(a) that a complete bandgap, highlighted by the dark blue region, opens up between the eighth band (brown) at point $W$ and the ninth band (red) at point $X$.

Since the upper edge of the photonic bandgap is limited by the $X$ point of the ninth band, and we empirically know that the upper (air) bands are usually more susceptible to modifications in the refractive index distribution, we suspect that shifting of the titania spheres toward the (100) direction, which corresponds to the $X$ point, can considerably alter the shape of the ninth band at that point, and result in the closing of the PBG. Prompted by this observation, we shift the inner spheres along (100) and calculate the resulting dispersion diagram. Figure 2(b) reports the result of such calculation with all optogeometric parameters being those of Fig. 2(a). We note that the previously complete bandgap is here altogether closed, with the perturbation of the ninth band being particularly noticeable at point $X$, as anticipated. The influence of the shifting of the spheres on the $X$ gap can also be seen by examining the field energy distributions at the $X$ point of the ninth band for the two switching states. The corresponding three-dimensional energy density distributions are illustrated at the right hand side of the band diagrams in Figs. 2(a) and 2(b). One can clearly observe that the field corresponding to the upper band is noticeably affected by the shifting of the dielectric spheres. Indeed, for the (100) switching state, a high intensity field (shown in red) develops and concentrates in a small area around the point where the titania sphere (yellow) touches the tin disulfide background (shown in green). This high intensity spot is completely absent in the (111) switching state, as shown in the right hand side of Fig. 2(a). From these results, we conclude that it is possible to completely open and close the photonic bandgap by changing the position of the titania spheres inside the air pores of the DIOPC structure.

Crucially, this scheme also allows for reasonable bandgap tuning and, furthermore, it turns out to be very stable with respect to variations of $R_{\mathrm{cyl}}$. The latter point is of great experimental interest since the control of $R_{\text {cyl }}$ during the fabrication process is an important issue. ${ }^{12}$ Figure 3(a) displays the variations with cylinder radius of the complete PBGs that occur upon moving the titania spheres along the (111) and (100) directions. In the present case, the same relatively small, sphere radius $R_{\mathrm{sp}}=0.13 a$ is chosen. This is to assure a suitable compromise between effectively manipulating the distribution of the fields when moving the spheres, which requires a sizeable radius, and an abundant room for the shifting itself, which requires smaller spheres. Figure 3(a) shows that for this choice of $R_{\mathrm{sp}}$ switching of the complete PBG is, in principle, possible for the entire range of cylinder radii $0<R_{\text {cyl }}<0.165 a$. The highlighted region indicates the experimentally interesting range of cylinder radii, since the maximum value that $R_{\text {cyl }}$ can assume in practice has to be smaller than $R_{\mathrm{sp}}$, in order for each sphere to be confined to its corresponding air pore and not fall through the cylinders. One can also observe that within the aforesaid range we do not have formation of a PBG for spheres shifted in the (100) direction. Accordingly, the investigated switching mechanism can be completely independent of $R_{\text {cyl }}$, provided that $R_{\text {cyl }}$ remains relatively small. Moreover, within the aforementioned range of cylinder radii, the switchable PBG exceeds $1.5 \%$ and assumes a maximum value of approximately $3.1 \%$, with larger values being achievable upon further optimization of the structure.

Finally, we analyzed the dependence of the complete PBG on the radius of the titania sphere, for fixed $R_{\text {cyl }}$ $=0.12 a$, and present the results in Fig. 3(b). One may observe that the curve which is associated with the (100) shift of the spheres drops off faster compared to the curve which corresponds to the (111) shift. Accordingly, there is a region of sphere radii, $0.126 a<R_{\mathrm{sp}}<0.178 a$, highlighted by the gray zone in Fig. 3(b), wherein we can switch from a complete PBG when the spheres are shifted along (111), to a closed PBG when the spheres are shifted along (100). Within this range the maximum switchable PBG is a sizeable $3.3 \%$.

In summary, we have presented a double-inverse-opal photonic crystal structure, comprised of a tin disulfide background and titania core spheres, which allows for dynamic PBG tuning. We have found that the shifting of the embedded titania spheres can substantially modify the optical properties of the structure, to the point of allowing for complete and robust PBG switching. The scheme is conceptually simple and elegant and, furthermore, there is compelling evidence that the fabrication process is reliable and steadfast. ${ }^{11,12}$ This method for achieving transparent/opaque switching of material aspect conceivably heralds a fresh PC design paradigm to be used in the realization of iridescent surfaces, switchable windows and in the dynamic control of optical properties.

We thank T. Ruhl, P. Spahn, C. Hermann, K. Boehringer and J. Allam for fruitful discussions, and J. Hamm for technical assistance. This work was partially supported by the Engineering and Physical Sciences Research Council (EPSRC), UK, and the German Federal Ministry of Research and Education within the project KODO.

${ }^{1}$ S. John, Phys. Rev. Lett. 58, 2486 (1987).

${ }^{2}$ E. Yablonovitch, Phys. Rev. Lett. 58, 2059 (1987).

${ }^{3}$ J. H. Holtz and S. A. Asher, Nature (London) 389, 829 (1997).

${ }^{4}$ Y. Y. Li, F. Cunin, J. R. Link, T. Gao, R. E. Betts, S. H. Reiver, V. Chin, S. N. Bhatia, and M. J. Sailor, Science 299, 2045 (2003).

${ }^{5}$ A. C. Arsenault, T. J. Clark, G. von Freymann, L. Cademartiri, R. Sapienza, J. Bertolotti, E. Vekris, S. Wong, V. Kitaev, I. Manners, R. Z. Wang, S. John, D. Wiersma, and G. A. Ozin, Nat. Mater. 5, 179 (2006).

${ }^{6}$ S. Nishimura, N. Abrams, B. A. Lewis, L. I. Halaoui, T. E. Mallouk, K. D. Benkstein, J. van de Lagemaat, and A. J. Frank, J. Am. Chem. Soc. 125, 6306 (2003).

${ }^{7}$ K. Yoshino, Y. Kawagishi, M. Ozaki, and M. Kose, Jpn. J. Appl. Phys., Part 2 38, L786 (1999).

${ }^{8}$ S. W. Leonard, H. M. van Driel, J. Schilling, and R. B. Wehrspohn, Phys. Rev. B 66, 161102 (2003).

${ }^{9}$ Y. A. Vlasov, M. O'Boyle, H. F. Hamann, and S. J. McNab, Nature (London) 483, 65 (2005).

${ }^{10}$ G. Mertens, T. Röder, H. Matthias, H. Marsmann, H.-S. R. Kitzerow, S. L. Schweizer, C. Jamois, R. B. Wehrspohn, and M. Neubert, Appl. Phys. Lett. 83, 3036 (2003).

${ }^{11}$ T. Ruhl, P. Spahn, C. Hermann, C. Jamois, and O. Hess, Adv. Funct. Mater. 16, 885 (2006).

${ }^{12}$ T. Ruhl, P. Spahn, H. Winkler, and G. P. Hellmann, Macromol. Chem. Phys. 205, 1385 (2004).

${ }^{13}$ S. G. Johnson and J. D. Joannopoulos, Opt. Express 8, 173 (2001). 